\title{
Model-based iterative approach to polarimetric radar rainfall estimation in presence of path attenuation
}

\author{
G. Vulpiani ${ }^{1}$, F. S. Marzano ${ }^{1}$, V. Chandrasekar ${ }^{2}$, and R. Uijlenhoet ${ }^{3}$ \\ ${ }^{1}$ Dipartimento di Ingegneria Elettrica - Centro di Eccellenza CETEMPS, Università dell'Aquila, Italy \\ ${ }^{2}$ Colorado State University, Fort Collins, Colorado, USA \\ ${ }^{3}$ Department of Environmental Sciences, Wageningen University, Wageningen, The Netherlands
}

Received: 17 November 2004 - Revised: 28 January 2005 - Accepted: 2 February 2005 - Published: 29 March 2005

\begin{abstract}
A new model-based iterative technique to correct for attenuation and differential attenuation and retrieve rain rate, based on a neural-network scheme and a differential phase constraint, is presented. Numerical simulations are used to investigate the efficiency and accuracy of this approach named NIPPER. The simulator is based on a T-matrix solution technique, while precipitation is characterized with respect to shape, raindrop size distribution and orientation. A sensitivity analysis is performed in order to evaluate the expected errors of this method. The performance of the proposed methodology on radar measurements is evaluated by using one-dimensional Gaussian shaped rain cell models and synthetic radar data derived from disdrometer measurements. Numerical results are discussed in order to evaluate the robustness of the proposed technique.
\end{abstract}

\section{Introduction}

Rainfall retrieval by using ground-based weather radar is achieving increased relevance in the evaluation of the hydrological cycle and in the monitoring of severe events. Nearly all European weather radars operate at $\mathrm{C}$ band, mainly due to cost constraints. However, at frequencies higher than $\mathrm{S}$ band, path attenuation effects due to rainfall can be significant and need to be compensated for quantitative estimation of rainrate. In this regard, dual-polarized weather radars represent a unique technological resource to mitigate this problem as shown in recent literature (e.g., Bringi and Chandrasekar, 2001).

Several approaches have been proposed to exploit polarimetric observables for rainfall estimation. Algorithms using the specific differential propagation phase $K_{d p}$ are immune to path attenuation effects (e.g., Zrnic and Ryzhkov, 1996). The specific differential propagation phase is the slope of the range profile of differential phase shift $\Phi_{d p}$, which can be estimated with an accuracy of few degrees. The iterative ap-

Correspondence to: G. Vulpiani

(g.vulpiani@aquila.infn.it) proaches (e.g., Hildebrand, 1978) for path attenuation correction, beginning from the closest (to the radar) range resolution volume and proceeding to farther (successive) resolution volumes, are known to be unstable. Besides, these methods generally assume a power law relation between reflectivity and specific attenuation and are sensitive to the radar calibration.

A significant improvement to these path-attenuation correction procedures is provided by using the total pathintegrated attenuation (PIA) as a constraint. This approach, originally proposed for spaceborne radar applications where the sea or land surface is generally assumed as a reference target, have been extended to ground-based polarimetric radar. Recently, the use of cumulative differential phase $\left(\Phi_{d p}\right)$ constraint to estimate the PIA and to correct the measured reflectivity $Z_{h h}$ and differential reflectivity $Z_{d r}$, proposed and evaluated by Testud et al. (2000) and Le Bouar et al. (2001) respectively, was improved by Bringi et al. (2001) through the use of a self-consistent scheme.

The objective of this paper is to introduce an alternative approach with $\Phi_{d p}$ utilized as a constraint to retrieve $Z_{h}$ and $Z_{d r}$ at $\mathrm{C}$ band in presence of significant path attenuation and, consequently, rainfall at ground. A model-based data set is used in an embedded neural network to train the retrieval algorithms. The neural network approach is applied in cascade to estimate rain rate from corrected polarimetric variables. Analysis of model data and numerical tests on synthetic radar data are discussed. The synthetic data are either created by a statistical generator or by spatial conversion of temporal series of disdrometer data.

\section{Polarimetric scattering model of rainfall}

A Gamma raindrop size distribution $(R S D)$, having the general form $N(D)=N_{0} D^{\mu} \exp (-\Lambda D)$ with $D$ the particle diameter and $N_{0}, \mu$ and $\Lambda R S D$ parameters, has been introduced in the literature to account for most of the variability occurring in the naturally observed $R S D$. 

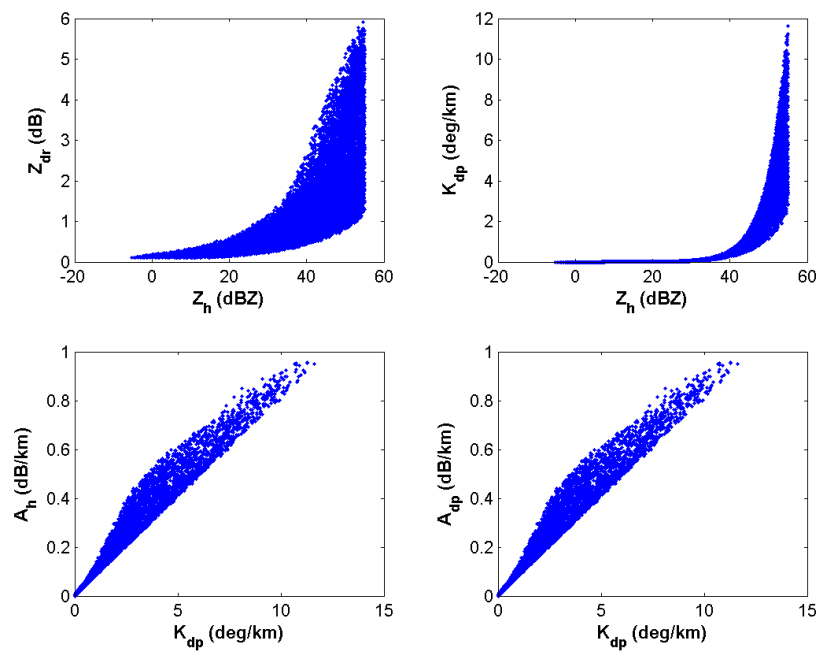

Fig. 1. Scatterplot of $Z_{d r}$ vs $Z_{h h}, K_{d p}$ vs $Z_{h h}, A_{h h}$ vs $K_{d p}$, and $A_{d p}$ vs $K_{d p}$ at $\mathrm{C}$ band. Amplitude variables are expressed in $\mathrm{dB}$.

The multiplication constant $N_{0}$, which has units depending on $\mu$ (i.e., $\left[\mathrm{mm}^{-1-\mu} \mathrm{m}^{-3}\right]$ ) is not physically meaningful when $\mu \neq 0$. In order to study the underlying shape of the $R S D$ for widely varying rainfall rates, the concept of normalization has been introduced by Willis (1984) and revisited by Chandrasekar and Bringi (1987), Testud et al. (2001) and Illingworth and Blackman (2002). The number of raindrops per unit volume per unit size can be written as:

$N(D)=N_{w} f(\mu)\left(\frac{D}{D_{0}}\right)^{\mu} \exp \left[-(3.67+\mu) \frac{D}{D_{0}}\right]$

where $f(\mu)$ is a function $\mu$ only, the parameter $D_{0}$ is the median volume drop diameter, $\mu$ is the shape of the drop spectrum, and $N_{w}\left[\mathrm{~mm}^{-1} \mathrm{~m}^{-3}\right]$ is a normalized drop concentration that can be calculated as function of liquid water content $\mathrm{W}$ and $D_{0}$ (e.g., Bringi and Chandrasekar, 2001).

The shape of a raindrop can be described by an oblate spheroid for which the equivalent volume diameter $D_{e}$ is related to the axis ratio $a / b$ by a relation which has been investigated by several authors. In this study we limited our attention to the relationship introduced by Pruppacher and Beard (1970) (named PB) and to the combination of those proposed by Andsager et al. (1999) and Chuang and Beard (1990) (named AB). Given a $R S D$, the rainfall intensity $R$ can be computed as a flux of raindrop volume at a terminal fall velocity $v(D)$, usually parameterized as a power law of D.

\subsection{Polarimetric radar variables}

The copolar radar reflectivity factors $Z_{h h}$ and $Z_{v v}\left[\mathrm{~mm}^{6} \mathrm{~m}^{-3}\right]$ at $\mathrm{H}$ and $\mathrm{V}$ polarization state, the cross-polar reflectivity factor $Z_{h v}$, the differential reflectivity $Z_{d r}$ and the linear depolarization ratio $L D R$ can be expressed as follows:

$Z_{h h, v v, h v}=\frac{\lambda^{4}}{\pi^{5}|K|^{2}}<4 \pi\left|S_{h h, v v, h v}^{b}(D)\right|^{2}>$
$Z_{d r}=\frac{Z_{h h}}{Z_{v v}}, \quad L D R_{v h}=\frac{Z_{v h}}{Z_{h h}}$

where $S_{h h, v v}$ and $S_{h v}([\mathrm{~mm}])$ are the backscattering co-polar and cross-polar components of the complex scattering matrix $\mathbf{S}$ of a raindrop, the angular brackets represent the ensemble average over the $R S D$. $K$ depends on the complex dielectric constant of water estimated as a function of wavelength $\lambda$ $([\mathrm{mm}])$ and temperature.

For a polarimetric radar, the specific differential phase shift $K_{d p}$, due to the forward propagation phase difference between $\mathrm{H}$ and $\mathrm{V}$ polarization and co-polar correlation coefficients $\rho_{h v}$ can be obtained in terms of the scattering matrix $\mathbf{S}$ as:

$K_{d p}=10^{-3} \frac{180}{\pi} \lambda \cdot \operatorname{Re}\left[<f_{h h}(D)-f_{v v}(D)>\right]$

$\rho_{h v}=\frac{<S_{v v} S_{h h}^{*}>}{\sqrt{<\left|S_{h h}\right|^{2}><\left|S_{v v}\right|^{2}>}}=\left|\rho_{h v}\right| e^{j \delta}$

where $f_{h h, v v}$ are the forward-scattering co-polar components of $\mathbf{S}$ and $\delta$ (in deg) is the volume backscattering differential phase. The specific attenuation $A_{h h}$ at $\mathrm{H}$ polarization and the differential attenuation $A_{d p}$ are finally defined as:

$A_{h h}=2 \cdot 10^{-3} \lambda \cdot \operatorname{Im}\left[<f_{h h}(D)>\right], \quad A_{d p}=A_{h h}-A_{v v}$

where specific attenuations are in $\mathrm{km}^{-1}$.

\subsection{Numerical examples at $\mathrm{C}$ band}

Once a $R S D$ is defined, the polarimetric radar parameters can be computed from the equations given in Sect. 2.1. Numerically computed forward scatter and backscatter amplitudes of raindrops for a given size are used to compute the radar parameters for a given RSD (Mishchenko, 2000). Computations are carried out at $\mathrm{C}$ band and the normalized Gamma $R S D$ is assumed for raindrop diameters between 0.6 and $8 \mathrm{~mm}$.

In order to generate a large set of model-based polarimetric variables, we adopted for $D_{0}$ and $\mu$ a uniform distribution inside the range proposed by Chandrasekar et al. (1987), that is $0.5 \leq D_{0} \leq 3.5 \mathrm{~mm}$ and $-1<\mu \leq 4$. As already mentioned, $N_{w}$ has been generated by assuming a random distribution of water content $W$ which results into a variability of rain rate from 0 to $300 \mathrm{~mm} / \mathrm{h}$. Temperatures of raindrops have been varied between $5^{\circ} \mathrm{C}$ and $30^{\circ} \mathrm{C}$ with a step of $5^{\circ} \mathrm{C}$. We are assuming here the most widely varying $R S D$ parameters without any correlation among them in order to ensure the training of the retrieval algorithm even in the most general conditions.

As an example of this randomly-generated polarimetric dataset, Fig. 1 shows the scatterplot of $Z_{d r}$ vs $Z_{h h}, K_{d p}$ vs $Z_{h h}, A_{h h}$ vs $K_{d p}$, and $A_{d p}$ vs $K_{d p}$ at C band. Amplitude variables are expressed in $\mathrm{dB}$. The values of $Z_{h h}$ are varied up to $55 \mathrm{dBZ}$. It is interesting to note the dominant linear correlation between the specific differential phase and the specific attenuations, even though a non negligible variance is appreciable for values of $K_{d p}$ larger than $5^{\circ} / \mathrm{km}$. Figure 2 shows 

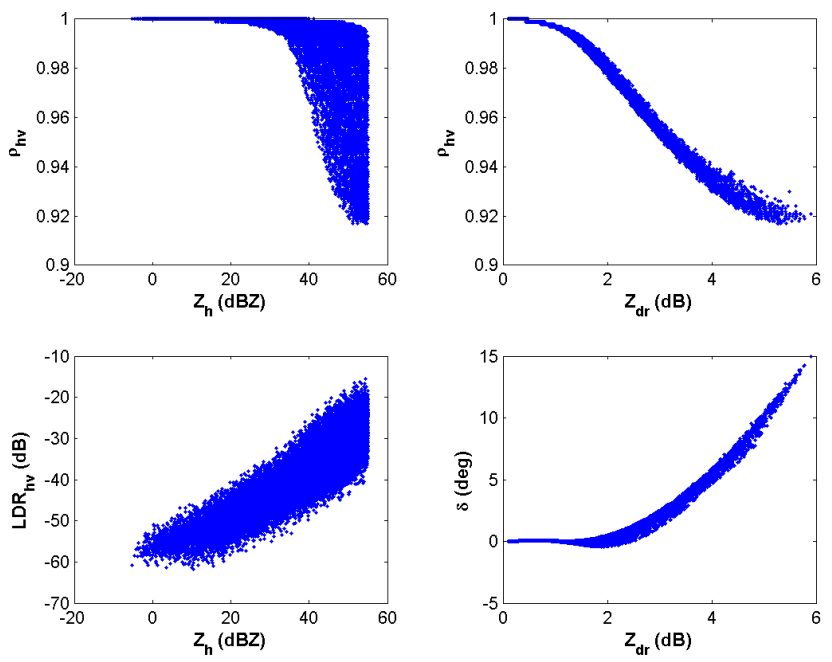

Fig. 2. As in Fig. 1, but for $\rho_{h v}$ vs $Z_{h h}, \rho_{h v}$ vs $Z_{d r}, L D R$ vs $Z_{h h}$, and $\delta$ vs $Z_{d r}$.

the scatterplot of $\rho_{h v}$ vs $Z_{h h}, \rho_{h v}$ vs $Z_{d r}, L D R$ vs $Z_{h h}$, and $\delta$ vs $Z_{d r}$. Note the non-linear correlation between $\rho_{h v}$ and $\delta$ with $Z_{d r}$ as shown by Scarchilli et al. (1993). Note that $L D R$ shows a negligible contribution of cross-polar backscattered power, while the values of $\rho_{h v}$ are always higher than 0.92 .

The correlation between the main polarimetric variables and the rainrate $R$ is illustrated in Fig. 3. This scatterplot is very instructive as it highlights the possible statistical relation and their expected accuracy for designing an inversion algorithm. From the figure, we note that, apart from $Z_{h h}$, $K_{d p}$ and $A_{h}$ show a relatively high correlation with $R$.

\section{Rainfall retrieval from polarimetric radar data}

The proposed retrieval technique, named Neural Iterative Polarimetric Precipitation Estimation by Radar (NIPPER) is illustrated in the next paragraphs. It consists of two steps in cascade where:

i) rain path attenuation is corrected by using an iterative scheme with the aid of a total path attenuation constraint and model-based neural-network estimates of the unknown parameters;

ii) a neural-network rain retrieval algorithm is applied to the polarimetric observable previously corrected within each single volume bin.

The main features of the NIPPER algorithm are that it does not assume any given analytical relation between the various polarimetric variables and it exploits the neural-network potential as a retrieval tool in a non linear context.

\subsection{Iterative correction of rain path attenuation}

Bringi et al. (1990) showed that $\Phi_{d p}$ is directly related to path integrated attenuation. It is possible to estimate the PIA from $\Phi_{d p}$ and use it to constrain the attenuation correction procedure (Testud et al., 2000). $A_{h}$ and $A_{d p}$ (both expressed
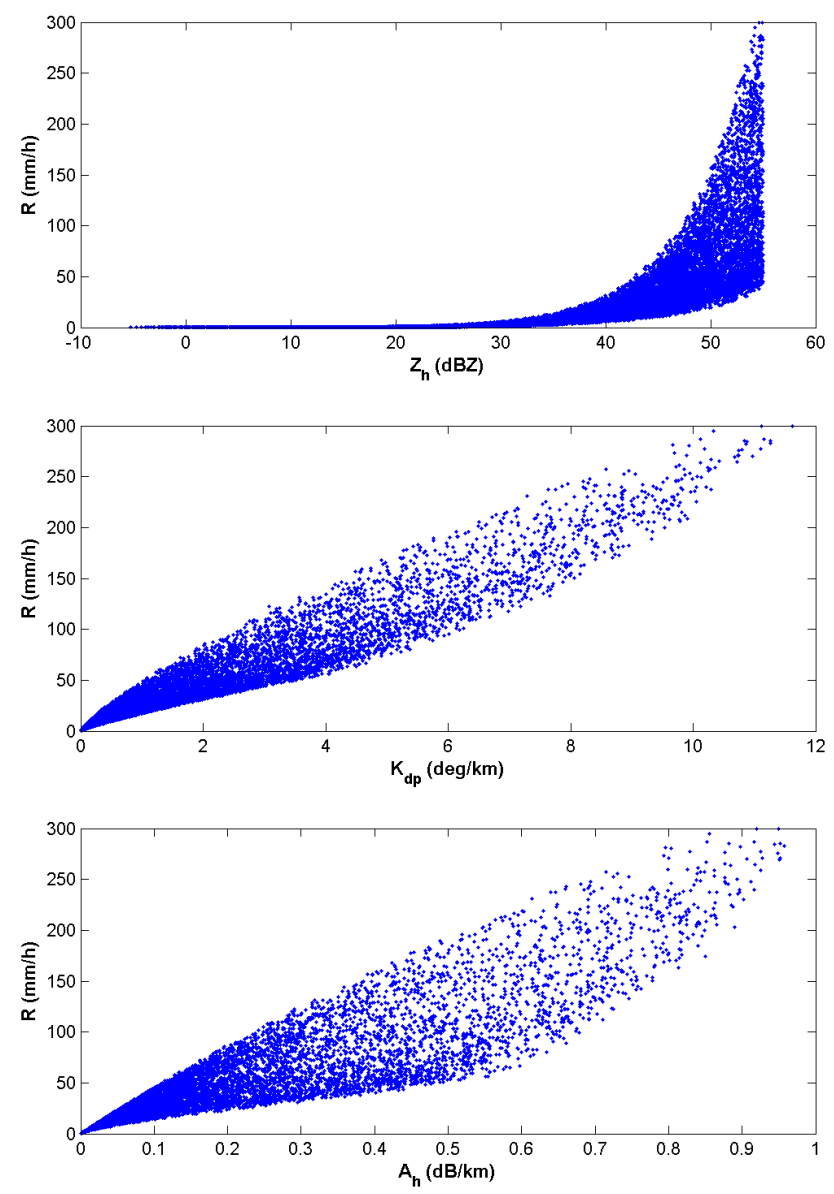

Fig. 3. As in Fig. 1, but for polarimetric variables vs. rainrate $R$.

in $\mathrm{dB} \mathrm{km}^{-1}$ ) are linearly related to $K_{d p}$ (in ${ }^{\circ} \mathrm{km}^{-1}$ ) which is the range derivative of $\Phi_{d p}$. Note that, before applying the attenuation correction scheme the differential phase shift $\Phi_{d p}$ must be filtered from the backscattering effects which are non negligible at C band (Scarchilli et al., 1993).

The proposed iterative algorithm is described in the following. The first step is the estimation of the path integrated attenuation $\operatorname{PIA}_{h}\left(r_{N}\right)$, and the path integrated differential attenuation $P I A_{d p}\left(r_{N}\right)$, at the farthest range $r_{N}$ by using the $\Phi_{d p}$ constraint. Therefore the corrected values of $Z_{h h}$ and $Z_{d r}$ are derived at the farthest range volume (here the $N^{\text {th }}$ ) by:

$Z_{h h, d r}^{C}\left(r_{N}\right)=Z_{h h, d r}^{m}\left(r_{N}\right)+2 P I A_{h h, d p}\left(r_{N}\right)$

where both reflectivities and PIA are expressed in $\mathrm{dB}$, while the superscripts $C$ and $m$ stand respectively for corrected and measured.

Using the corrected values of $Z_{h h}$ and $Z_{d r}$, it is possible to estimate the specific attenuation (and the specific differential attenuation), through a neural network, at the $N^{\text {th }}$ range volume by means of:

$A_{h h, d r}\left(r_{N}\right)=N N_{A}\left(Z_{h h}^{C}\left(r_{N}\right), Z_{d r}^{C}\left(r_{N}\right)\right)$ 

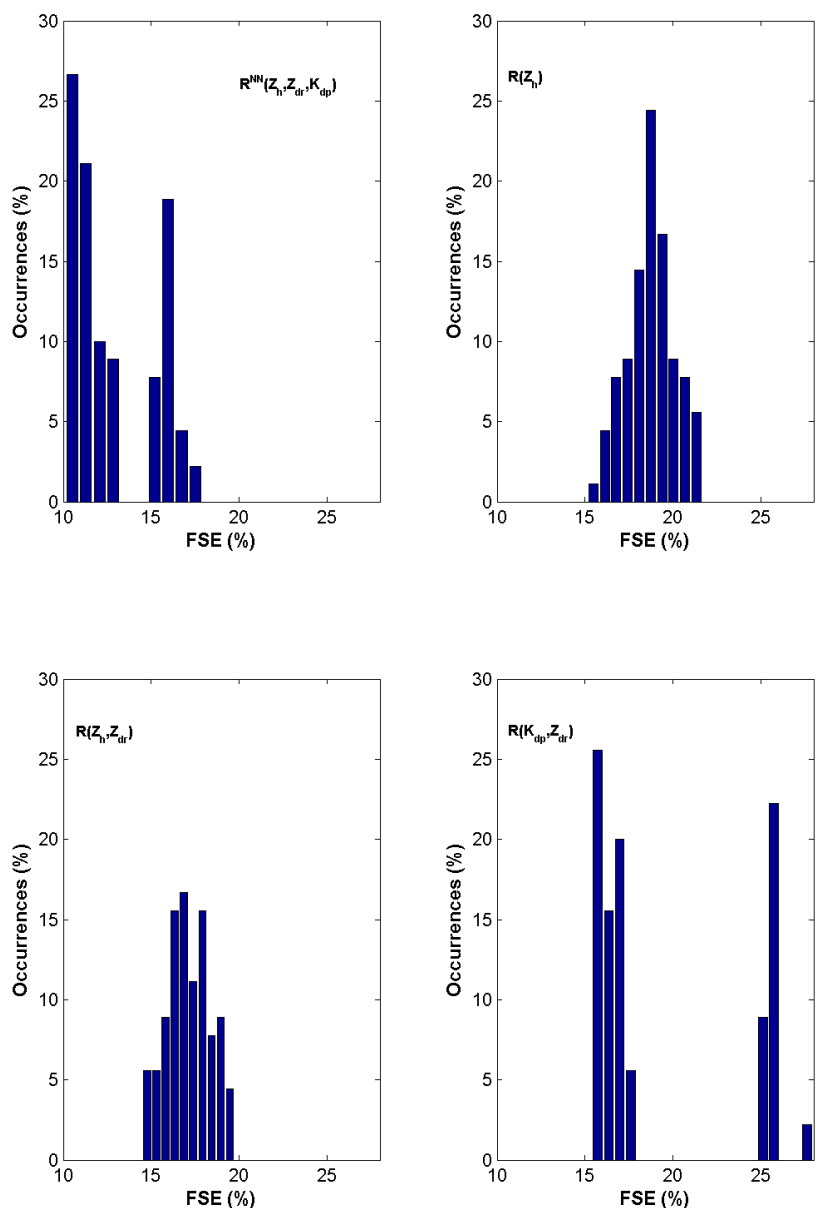

Fig. 4. Performance of NIPPER algorithm in terms of the histogram of fractional standard error (FSE) calculated for each range profile realization for 4 rain retrieval algorithms applied to corrected polarimetric observables, i.e. besides $R\left(Z_{h h}^{C}\right), R\left(Z_{h h}^{C}, Z_{d r}^{C}\right), R\left(Z_{d r}^{C}\right.$, $\left.K_{d p}^{C}\right)$ and $R_{N N}\left(Z_{h h}^{C}, Z_{d r}^{C}, K_{d p}^{C}\right)$.

where $N N_{A}$ is a Neural Network functional used for the specific attenuation (differential attenuation) estimation. As a consequence we can estimate the $P I A$ at the $(N-1)^{\text {th }}$ range bin from:

$P I A_{h h, d p}\left(r_{N-1}\right)=P I A_{h h, d p}\left(r_{N}\right)-A_{h h, d r}\left(r_{N}\right) \cdot \Delta r$

where $\Delta r$ is the bin range resolution, while the corrected values of reflectivity and differential reflectivity are

$Z_{h h, d r}^{C}\left(r_{N-1}\right)=Z_{h h, d r}^{m}\left(r_{N-1}\right)+2 P I A_{h h, d p}\left(r_{N-1}\right)$

Generalizing (9) and (10) for the $K^{\text {th }}$ range volume, we can write:

$$
\begin{aligned}
& P I A_{h h, d p}\left(r_{K}\right)=P I A_{h h, d p}\left(r_{N}\right)-\int_{\left(r_{K}-1\right) \Delta r}^{r_{K} \Delta r} A_{h h, d r}(s) \cdot d s \\
& Z_{h h, d r}^{C}\left(r_{K}\right)=Z_{h h, d r}^{m}\left(r_{K}\right)+2 P I A_{h h, d p}\left(r_{K}\right)
\end{aligned}
$$

Through (11) and (12) it is possible to iteratively correct the profiles of $Z_{h h}$ and $Z_{d r}$. At each range volume a control check on $\Phi_{d p}$ filtering is performed estimating $\delta$ by means of corrected variables using an ad hoc neural-network algorithm (Vulpiani et al., 2003).

\subsection{Rain rate retrieval}

One of the applications of polarimetric radars is the possibility to use different algorithms in order to estimate the rainfall rate. Apart from $R\left(Z_{h h}\right)$, polarization diversity allows to employ the two-parameter algorithms $R\left(Z_{h h}, Z_{d r}\right)$ and $R\left(Z_{d r}, K_{d p}\right)$ as well as $R\left(K_{d p}\right)$. The algorithms using reflectivity and differential reflectivity are affected by absolute and differential radar calibration errors. On the other hand, those using the $K_{d p}$ have the impact of the scheme adopted to derive it from $\Phi_{d p}$ which could be contaminated by the backscattering differential phase.

The rainfall estimator proposed in this work, indicated as $R_{N N}\left(Z_{h}, Z_{d r}, K_{d p}\right)$, is based on a feed-forward neural network with a back-propagation learning algorithm and uses the retrieved corrected profiles of $Z_{h}, Z_{d r}$, and $K_{d p}$. In a formal way, we can write:

$R_{N N}=N N_{R}\left(Z_{h h}^{C}, Z_{d r}^{C}, K_{d p}^{C}\right)$

where $N N_{R}$ is again a Neural Network functional used for the rain rate estimation.

\section{Numerical tests on synthetic radar data}

In this subsection we analyze the numerical results obtained applying the proposed NIPPER inversion technique to the synthetic radar data sets assuming that the radar is well calibrated. The evaluation of the radar system bias effects on the retrieval performance is discussed in Vulpiani et al. (2004).

\subsection{Synthetic radar data from statistical generator}

As already noted when discussing (1), the intensity of rain events can be characterized using the liquid water content $W$ or the rainfall rate $R$. In this work we adopted a double Gaussian-shaped range profile of liquid water content $W$ in $\left[\mathrm{g} \mathrm{m}^{-3}\right]$ in order to derive the $N_{w}$ range profile and the randomly generated values of $D_{0}$ and $\mu$ as inputs. For each range bin of this synthetic profile, the axis ratio model is selected randomly between the $\mathrm{PB}$ and $\mathrm{AB}$ relationships, according to a uniform distribution. The dielectric constant, is dependent on temperature and has been assumed constant along the profile. A random noise on simulated polarimetric variables has been introduced to realistically reproduce the measurebles, resulting in a $1 \mathrm{~dB}$ noise for $Z_{h h}, 0.3 \mathrm{~dB}$ noise for $Z_{d r}$ and $2^{\circ}$ noise for $\Phi_{d p}$. One hundred range profiles have been generated in this study.

As a quality metric of the retrieved rain rate, we have considered the fractional standard error (FSE), which is the root mean square error normalized to the mean true value, averaged along the range profile.

Figure 4 shows the performance of the NIPPER algorithm in terms of the histogram of FSE calculated for each range 

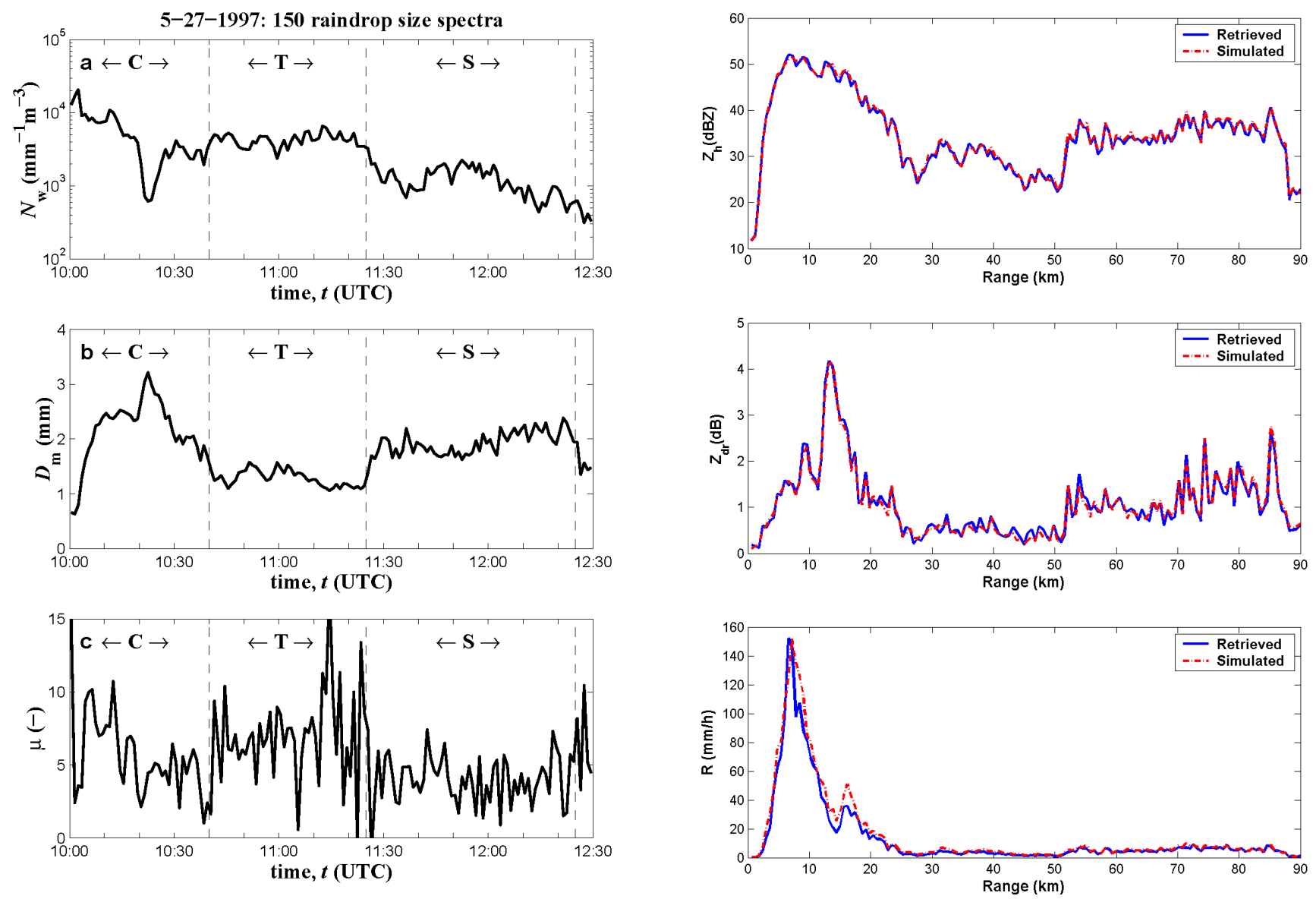

Fig. 5. Time series of estimated $N_{w}, D_{m}$ and $\mu$ from disdrometer data, fitted to the normalised Gamma $R S D$ as in (1), acquired in Northern Mississippi (Uijlenhoet et al., 2003).

profile realization for the four rain retrieval algorithms applied to corrected measurements, namely $R\left(Z_{h h}^{C}\right), R\left(Z_{h h}^{C}\right.$, $\left.Z_{d r}^{C}\right), R\left(Z_{d r}^{C}, K_{d p}^{C}\right)$ and $R_{N N}\left(Z_{h h}^{C}, Z_{d r}^{C}, K_{d p}^{C}\right)$. It is worth mentioning that the first three are the "best" parametric algorithms.

The comparison clearly shows how the neural-network approach is more accurate in the reconstruction of the rain with errors always below $20 \%$.

\subsection{Synthetic radar data from disdrometer measurements}

The ZPHI algorithm is based on the hypothesis that the exponent $\beta$, characterising the relation between reflectivity and attenuation, is constant and $N_{w}$ is a "local" variable that is reasonably constant at a scale of about $10 \mathrm{~km}$ (Testud et al., 2000). While scattering simulations have demonstrated that the first assumption is reasonable at C-band, the second hypothesis depends on the properties of prevailing precipitation.

See for example a case study for a squall-line system passing over a watershed in northern Mississippi presented by Uijlenhoet et al. (2003). Figure 5 shows the time series of

Fig. 6. Behavior of simulated $Z_{h h}, Z_{d r}$, and $R$ derived from Fig. 5 by assuming a storm velocity of $10 \mathrm{~m} / \mathrm{s}$. Retrieved quantities, derived from NIPPER, are also plotted.

estimated $N_{w}, D_{m}$ and $\mu$ from disdrometer data fitted to the normalised Gamma $R S D$ as in (1). Note that $D_{m}$ is the massweighted mean diameter closely related to the median volume diameter $D_{0}$ (Bringi and Chandrasekar, 2001). Convective, transition and stratiform regions are denoted by "C", "T" and "S", respectively.

As shown in Fig. 5, during this event $N_{w}$ is characterized by significant variations not only in the transition phase $(\mathrm{T})$ but also in the pure convective phase.

In order to convert the disdrometer time series into range profile, an average fixed storm velocity of $10 \mathrm{~m} / \mathrm{s}$ has been assumed. Analyzing the relative behaviour of the simulated $R, Z_{h h}$ and $Z_{d r}$, shown in Fig. 6, it can be argued that the observed jump was due to a sequence of contrasting regimes within the convective phase of the squall-line system. To a certain extent, the assumption of "moderate" variability inside a specific rain regime has not a general validity and should be carefully used. The use of analytical solutions to path attenuation correction could not be suitable under these conditions, given the dependence from $N_{w}$. This is the reason why the NIPPER algorithm can show some advantages in circumstances where there is a significant $N_{w}$ variability. 

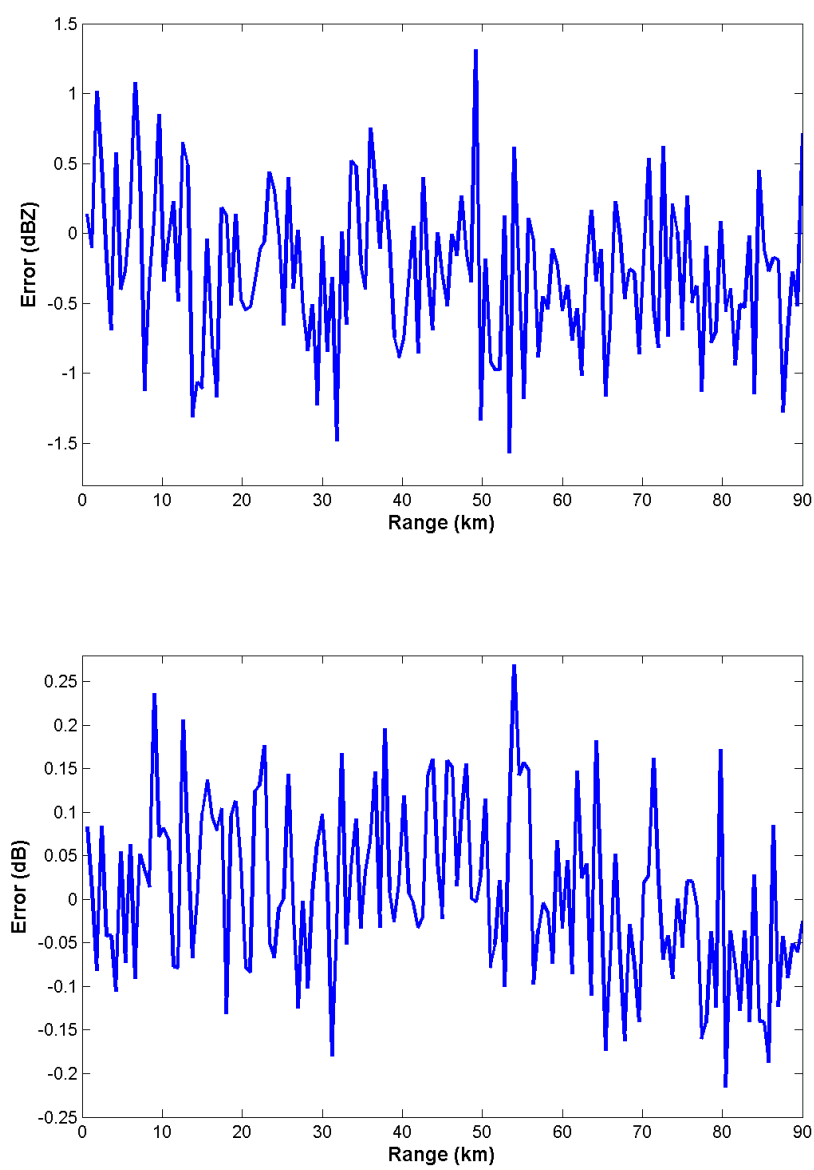

Fig. 7. Errors in $\mathrm{dB}$ for the reconstruction of $Z_{h h}$ and $Z_{d r}$ as in Fig. 6.

In Fig. 6 the comparison of the simulated "true" values with path-attenuation corrected $Z_{h h}^{C}$ and $Z_{d r}^{C}$, and retrieved $R_{N N}$ by NIPPER is also shown. Figure 7 shows the errors of the $Z_{h h}$ and $Z_{d r}$ reconstruction at each range bin.

Both figures show a fairly good overall behaviour of retrievals. Errors on rain rate retrievals are less than $20 \%$ even for peaks of rain rate above $100 \mathrm{~mm} / \mathrm{h}$, while the reconstruction errors of the corrected $Z_{h h}$ and $Z_{d r}$ are less than $1 \mathrm{~dB}$ and $0.1 \mathrm{~dB}$ on average, respectively.

\section{Conclusions}

A new iterative technique, named NIPPER, to correct for attenuation and differential attenuation, based on a neuralnetwork scheme and a differential phase constraint, and to retrieve rain rate from polarimetric radar data has been presented. The main features of the NIPPER algorithm are that it is a non-parametric transformation and it exploits the neural-network potential as a retrieval tool.

Numerical simulations have been used to investigate the performance of this approach. A retrieval test analysis has been performed in order to evaluate the expected errors of this method.
The performance of the proposed methodology on radar measurements has been evaluated by using one-dimensional Gaussian rain cell models and synthetic radar data derived from disdrometer measurements. Numerical results have demonstrated the potential and robustness of the proposed technique.

Acknowledgements. This work has been partially funded by European project Risk-AWARE within INTERREG-IIIB-CADSES and by the Italian Ministry of University and Research (MIUR). One of the authors (VC) acknowledges support from the NSF-ERC program (CASA-ERC-0313747). The author R. U. is financially supported by the Netherlands Organization for Scientific Research (NWO) through a Vernieuwingsimpuls/VIDI grant (Project 016.021.003). The authors thank M. Steiner (Princeton University) who was responsible for the collection of the disdrometer data used in Fig. 5.

Edited by: L. Ferraris

Reviewed by: anonymous referees

\section{References}

Andsager, K., Beard, K. V., and Laird, N. F.: Laboratory measurements of axis ratios for large rain drops, J. Atmos. Sci., 56, 26732683, 1999.

Bringi, V. N., Chandrasekar, V., Balakrishnan, N., and Zrnic, D. S.: An examination of propagation effects in rainfall on radar measurements at microwave frequencies, J. Atmos. Ocean. Technol., 7, 829-840, 1990.

Bringi, V. N., Keenan, T. D., and Chandrasekar, V.: Correcting Cband radar reflectivity and differential refelctivity data for rain attenuation: a self-consistent method with constraints, IEEE Trans. Geosci. Remote Sensing, 39, 1906-1915, 2001.

Bringi, V. N. and Chandrasekar, V.: Polarimetric doppler weather radar, Cambridge University Press, 2001.

Bringi, V. N., Chandrasekar, V., Hubbert, J., Gorgucci, E., Randeu, W. L., and Schoenhuber, M.: Raindrop size distribution in different climatic regimes from disdrometer and dual-polarized radar analysis, J. Atmos. Sci., 60, 354-365, 2002.

Chandrasekar, V. and Bringi, V. N.: Simulation of radar reflectivity and surface measurements of rainfall, J. Atmos. Oceanic Technol., 4, 464-478, 1987.

Chuang, C. and Beard, K. V.: A numerical model for the equilibrium shape of electrified raindrops, J. Atmos. Sci., 19, 1374 1389, 1990.

Gorgucci, E., Scharchilli, G., and Chandrasekar, V.: A procedure to calibrate multiparameter weather radar using properties of the rain medium, IEEE Trans. Geosci. Remote Sens., 37, 269-276, 1999.

Hildebrand, P. H.: Iterative correction for attenuation of $5 \mathrm{~cm}$ radar in rain, J. Appl. Meteor., 17, 508-514, 1978.

Hubbert, J. and Bringi, V.: An iterative filtering technique for the analysis of copolar differential phase and dual-frequency radar measurements, J. Atmos. Ocean. Technol., 15, 643-648, 1995.

Illingworth, A. J. and Blackman, T. M.: The need to represent raindrop size spectra as normalized gamma distributions for the interpretation of polarization radar observations, J. Appl. Meteor., 41, 1578-1590, 2002. 
Jameson, A. R.: The effect of temperature on attenuation-correction schemes in rain using polarization propagation differential phase shift, J. Appl. Meteor, 31, 1106-1118, 1992.

Kozu, T. and Nakamura, K.: Rainfall parameter estimation from dual-radar measurements combining reflectivity profile and pathintegrated attenuation, J. Atmos. Oceanic Technol., 8, 259-270, 1991.

Le Bouar, E., Testud, J., and Keenan, T. D.: Validation of the rain profiling algorithm ZPHI from the C-band polarimetric weather radar in Darwin, J. Atmos. Ocean. Technol., 18, 1819-1837, 2001.

Mishchenko, M. I.: Calculation of the amplitude matrix for a nonspherical particle in a fixed orientation, Appl. Opt., 39, 10261031, 2000

Pruppacher, H. R. and Beard, K. V.: A wind tunnel investigation of the internal circulation and shape of water drops falling at terminal velocity in air, Q. J. R. Meteorol. Soc., 96, 247-256, 1970.

Scarchilli, G., Gorgucci, E., Chandrasekar, V., and Seliga, T. A.: Rainfall estimation using polarimetric techniques at C-band frequencies, J. Appl. Meteor., 32, 1150-1160, 1993.
Testud, J., Le Bouar, E., Obligis, E., and Ali-Mehenni, M.: The rain profiling algorithm applied to polarimetric weather radar, J. Atmos. Ocean. Technol., 17, 332-356, 2000.

Testud, J., Oury, S., Black, R. A., Amayenc, P., and Dou, X.: The concept of "normalized" distribution to describe raindrop spectra: A tool for cloud physics and cloud remote sensing, J. Appl. Meteor., 40, 1118-1140, 2001.

Uijlenhoet, R., Steiner, M., and Smith, J. A.: Variability of raindrop size distributions in a squall line and implications for radar rainfall estimation, J. Hydrometeor., 4, 43-61, 2003.

Vulpiani, G., Picciotti, E., Ferrauto, G., and Marzano, F. S.: Sensitivity Analysis of Self-Consistent Polarimetric Rain Retrieval to C-Band Radar Variables, Proc. of IGARSS03, Toulouse, 2003.

Vulpiani, G., Marzano, F. S., Ferrauto, G., Picciotti, E., and Chandrasekar, V.: C-band dual polarization radar retrieval of rainfall: application of an iterative technique with embedded neural network, Proc. of ERAD-04, Visby (SW), 386-392, 2004.

Willis, P. T.: Functional fit to some observed drop size distributions and parametrization of rain, J. Atmos. Sci., 41, 1648-1661, 1984.

Zrnic, D. S. and Ryzhkov, A.: Advantages of rain measurements using specific differential phase, J. Atmos. Ocean. Technol., 13, 454-464, 1996. 